\title{
Semi-parametric risk prediction models for recurrent cardiovascular events in the LIPID study
}

\author{
Jisheng Cui ${ }^{1,2^{*}}$, Andrew Forbes ${ }^{2}$, Adrienne Kirby ${ }^{3}$, lan Marschner ${ }^{3,4}$, John Simes $^{3}$, David Hunt ${ }^{2}$, Malcolm West ${ }^{5}$, \\ Andrew Tonkin ${ }^{2}$
}

\begin{abstract}
Background: Traditional methods for analyzing clinical and epidemiological cohort study data have been focused on the first occurrence of a health outcome. However, in many situations, recurrent event data are frequently observed. It is inefficient to use methods for the analysis of first events to analyse recurrent event data.

Methods: We applied several semi-parametric proportional hazards models to analyze the risk of recurrent myocardial infarction (MI) events based on data from a very large randomized placebo-controlled trial of cholesterol-lowering drug. The backward selection procedure was used to select the significant risk factors in a model. The best fitting model was selected using the log-likelihood ratio test, Akaike Information and Bayesian Information Criteria.
\end{abstract}

Results: A total of 8557 persons were included in the LIPID study. Risk factors such as age, smoking status, total cholesterol and high density lipoprotein cholesterol levels, qualifying event for the acute coronary syndrome, revascularization, history of stroke or diabetes, angina grade and treatment with pravastatin were significant for development of both first and subsequent $\mathrm{Ml}$ events. No significant difference was found for the effects of these risk factors between the first and subsequent $\mathrm{Ml}$ events. The significant risk factors selected in this study were the same as those selected by the parametric conditional frailty model. Estimates of the relative risks and 95\% confidence intervals were also similar between these two methods.

Conclusions: Our study shows the usefulness and convenience of the semi-parametric proportional hazards models for the analysis of recurrent event data, especially in estimation of regression coefficients and cumulative risks.

\section{Background}

Many clinical and epidemiological cohort studies involve health outcomes that a participant of the study can experience several times during the follow-up period. Such outcomes are often termed recurrent or repeated events [1,2]. For example, transient ischemic attacks can occur repeatedly among patients with cerebrovascular disease [3]. A person who is infected with HIV may experience several opportunistic infections in HIV/AIDS clinical trials [4]. However, traditional statistical methods for the analysis of cohort study data have been focused on the first occurrence of an outcome [5]. It is reasonable to assume that the first occurrences of an

\footnotetext{
* Correspondence: jisheng.cui@deakin.edu.au

'World Health Organization Collaborating Centre for Obesity Prevention, Deakin University, Melbourne, Australia
}

(c) 2010 Cui et al; licensee BioMed Central Ltd. This is an Open Access article distributed under the terms of the Creative Commons Attribution License (http://creativecommons.org/licenses/by/2.0), which permits unrestricted use, distribution, and reproduction in any medium, provided the original work is properly cited.

outcome among different individuals are independent, which is an important assumption in modelling these events.

However, the methods for the analysis of first events are inefficient in the analysis of recurrent event data because of the possible correlation among recurrent outcomes in an individual. An individual may be prone to develop more recurrent events or the time interval between these events may be shorter than others in the study depending on the characteristics of the individual. Therefore, the assumption of independence of the event times in the first event analysis method is often violated for recurrent event data. Updated analytical methods are needed to account for the dependence of the repeated measurements in a person during follow-up study.

Various statistical methods have been proposed for the analysis of recurrent event data [6-9]. The conditional 
models usually define different risk sets for different recurrent events using stratification techniques $[8,10]$. The gap time between two consecutive recurrent events is usually used in a conditional model. The conditional model can be used to estimate the rate of a subsequent event given an individual has already had such an event during follow-up. On the other hand, Cox-type regression models have been considered by Prentice et al [11] and Andersen and Gill [12] in their seminal studies in this area. Wei et al [13] proposed a novel semi-parametric proportional hazards model and Lin [14] presented general theory for this method. Semi-parametric models have further been updated and applied by Pepe and Cai [15] for time-dependent covariates, by Lawless and Nadeau [16] for a robust method, and by Glidden and Vittinghoff [17] for analyzing multicenter clinical trial data. Wang and Chang [18] clarified two sampling designs in a longitudinal study and devoted their method to the design where the initial occurrence of an event is used as the enrolment criterion and repeated occurrences of the same event are observed during follow-up. Pena et al [19] and Pena et al [20] also discussed the general class of semi-parametric models for recurrent events. Metcalfe and Thompson [21] reviewed and evaluated the semi-parametric model method in the analysis of recurrent event data and concluded that the application of this method to recurrent event data is justified.

The aim of this study was to apply several semi-parametric models to the analysis of recurrent myocardial infarction (MI) events in a large randomized placebocontrolled clinical trial in Australia and New Zealand $[22,23]$. We focused on investigating the significant risk factors for both the first and recurrent MI events during follow-up and examining whether there was significant difference in their effects for the first and recurrent MI events.

\section{Methods}

\section{The LIPID study}

The Long-term Intervention with Pravastatin in Ischaemic Disease (LIPID) study [22-24] was a double-blinded randomized placebo-controlled trial of a cholesterollowering drug, pravastatin ( $40 \mathrm{mg}$ daily). The study was undertaken in 9014 patients who were 31 to 75 years of age at the time of randomization. They had a history of myocardial infarction or hospitalization for unstable angina 3-36 months previously and initial plasma total cholesterol levels of 155 to $271 \mathrm{mg}$ per deciliter (4.0 $7.0 \mathrm{mmol} /$ liter $)$ and triglyceride levels $<445 \mathrm{mg} / \mathrm{dl}(5$ $\mathrm{mmol} / \mathrm{L}$ ). Patients were followed up for a median of 6.0 years between 1990 and 1997. In the present analysis, data from the 8557 patients (4286 randomized to receive pravastatin and 4271 to receive placebo) who did not have any missing baseline information were used, which is consistent with a previous study [24]. The primary pre-specified endpoint in the LIPID study was coronary heart disease (CHD)-related death and for secondary analyses, a composite of CHD death or non-fatal myocardial infarction. Details of the study design have been published previously [22,23].

The outcome variables in this present analysis were the recurrence of an MI event during follow-up and the time from randomization to each MI event. Explanatory variables included gender ( 1 for males and 0 for females), age at randomization (centered at 60 years for ease of computing the baseline hazard rate), smoking status ( 1 for smokers and 0 for non-smokers), total serum cholesterol (centered at $5.0 \mathrm{mmol} / \mathrm{l})$, high density lipoprotein (HDL)-cholesterol (centered at $1.0 \mathrm{mmol} / \mathrm{l}$ ), treatment group ( 1 for pravastatin assignment and 0 for placebo), qualifying acute coronary syndrome (ACS) at baseline (unstable angina, single MI, or multiple MI before randomization), coronary revascularization (never, before the qualifying event, or after the qualifying event but before randomization), dyspnea grade $(0$ for NYHA < III and 1 for NYHA $\geq$ III) based on New York Heart Association definition [25], angina grade $(0$ for CCVS $<$ III and 1 for CCVS $\geq$ III) based on the Canadian Cardiovascular Society definition [26], duration of angina ( 0 for $\leq 5$ years and 1 for $>5$ years), use of aspirin ( 0 for no and 1 for yes), country of residence (Australia or New Zealand), and history of stroke, diabetes mellitus or hypertension. Interactions between these risk factors were also examined in the analysis.

The following multivariate regression models were fitted to the data to incorporate all the above mentioned explanatory variables. The backward selection method was used to select the final model which includes risk factors which were statistically significant at the 0.05 level.

\section{Semi-parametric model}

The semi-parametric model considered in this article is an extension of the Cox proportional hazards model [5]. Suppose there are $n$ participants in the study. For the $i$ th person $(i=1,2, \ldots, n)$, the model is of the following form

$$
h_{i}^{(k)}\left(t \mid x_{i}^{(k)}\right)=h_{0 i}^{(k)}(t) \exp \left[x_{i} \beta^{(k)}\right]
$$

where $h_{i}^{(k)}(t)$ is the general hazard function and $h_{0 i}^{(k)}(t)$ is the baseline hazard function at time $t$ for the $k^{\text {th }}$ recurrent event $(k=1, \ldots, K)$. The analysis time $t$ is calculated from the date of randomization to the date of each recurrent MI event for each person in the study. Both the risk factor $x$ and the associated regression 
coefficient $\beta^{(k)}$ are $(p+1)$-dimensional vectors, where $p$ is the number of covariates in the model. Parameter $\beta^{(k)}$ is estimated using the partial likelihood method [27]. The advantage of using the semi-parametric model is that it does not require explicit specification of the dependence structure between the times of recurrent events of each person; instead it uses the robust Huber/White sandwich estimator to obtain the variance of the estimated regression coefficients [28-30]. The robust variance estimator can be easily calculated using most of the standard statistical software packages. The cumulative risk of a specific MI event after randomization can be calculated by

$$
F_{i}^{(k)}(t)=1-\exp \left[-H_{i}^{(k)}(t)\right] .
$$

where $H_{i}^{(k)}(t)$ is the cumulative hazard by time $t$ [31]. The censoring mechanism was assumed to be independent of the recurrent event process, as it is in most standard time-to-event analyses. A person was censored if he/she died of non-cardiovascular causes, or was lost to follow-up or was alive at the end of the study. Therefore, a person might have several recurrent MI events, or might not experience any MI event before being censored. Multiple data records might be observed for each person for possible occurrence of an MI event or occurrence of censoring. However, the occurrence of an MI was assumed not to influence the occurrence of censoring, and vice versa, in the present analysis.

\section{Model comparison}

Several semi-parametric models were fitted to the LIPID study data. Model 1 assumes that the covariate effect $\beta^{(k)}$ and the baseline hazard rate $h_{0}^{(k)}(t)$ were different for the first and subsequent MI events. Model 2 assumes that the covariate effects $\beta^{(k)}$ were the same for the first and subsequent MI events but the baseline hazard rates $h_{0}^{(k)}(t)$ were different for these recurrent events. Model 3 assumes that the covariate effects $\beta^{(k)}$ were different for the recurrent events but the baseline hazard rates $h_{0}^{(k)}(t)$ were the same. Model 4 assumes that both the covariate effects $\beta^{(k)}$ and the baseline hazard rates $h_{0}^{(k)}(t)$ were the same for the recurrent events.

\section{Model fitting}

Appropriate data formats were prepared for fitting a semi-parametric model. No matter how many observed data records that a person can have, a total of $K$ records were created for each person in the study, where $K$ was the maximum number of recurrent events that were experienced by all participants in the study. For a person who had an MI event at time $t$, the value of the corresponding outcome was 1 for this time point. After the time of the final MI event, the value of the outcome was assumed to be 0 in order to make a total of $\mathrm{K}$ data records. In addition, an indicator variable was created for each data record of a person in the study. Details concerning preparation of appropriate data formats can be found in Therneau and Grambsch [8]. The statistical analyses in this article were conducted using Stata software version 10 [32], which allows semi-parametric model analysis to be conducted easily with no specialized programming required. These analyses were based on the intention-to-treat principle where an individual was assumed to take the treatment that was assigned to him or her at the time of randomization [33].

\section{Results}

\section{Follow-up in the LIPID Study}

Table 1 shows the number of MI events and the outcome status of participants at the end of the study. A total of $870 \mathrm{MI}$ events occurred in 745 patients (an average of 1.2 events per person) during follow-up, of whom 313 had been randomized to receive pravastatin and 432 to receive placebo. In all, 7.3\% (313/4286) of the persons who were assigned to receive pravastatin and $10.1 \%(432 / 4271)$ assigned to placebo had at least one MI event during follow-up. The former proportion was significantly lower than the latter $(z=4.6, p<$ 0.001 ). The number of persons who had more than one MI event during follow-up was generally low. About $0.9 \%(37 / 4286)$ of the persons in the pravastatin group and $1.3 \%(56 / 4271)$ in the placebo group had more than one MI event during follow-up. Because of the small numbers of participants who had more than two MI events in this study, the following analyses were focused on the first two MI events, i.e. we assumed $K=2$ in model (1).

Table 1 Number of myocardial infarctions and outcome status for the participants in the LIPID study

\begin{tabular}{cll}
\hline Variable & \multicolumn{2}{c}{ Treatment } \\
\cline { 2 - 3 } & Pravastatin & Placebo \\
\hline Number of Ml events & 3973 & 3839 \\
0 & 276 & 376 \\
1 & 31 & 39 \\
2 & 3 & 13 \\
3 & 2 & 3 \\
4 & 1 & 1 \\
5 & & \\
Outcome status & 470 & 592 \\
Died & 236 & 305 \\
Alive and had an Ml event & 3580 & 3374 \\
Alive but no Ml event &
\end{tabular}

$\mathrm{MI}=$ myocardial infarction 
Table 1 also shows that a total of 1062 patients ( 470 assigned pravastatin and 592 placebo) died during follow-up. Another 541 patients (236 assigned pravastatin, 305 placebo) experienced at least one MI event during follow-up and were still alive at the end of the study. The other 6954 patients (3580 assigned pravastatin, 3374 placebo) did not experience any MI event during follow-up and were still alive at the end of the study.

\section{Model comparison}

Different semi-parametric models are compared in Table 2. The same covariates were selected by all 4 models, which include age, smoking status, total cholesterol and HDL cholesterol levels, qualifying ACS event at baseline, coronary revascularization, history of stroke or diabetes, angina grade and treatment with pravastatin. Model 2 that has the same effect for all recurrent events but with different baseline hazard functions had the smallest AIC and BIC values, suggesting that this model fitted the data better than other three models. Model 1 that has different effects for the recurrent events and different baseline hazard functions had the second smallest AIC and BIC values. The likelihood ratio test showed that there was no significant difference between model 1 and model $2\left(\chi^{2}=\right.$ 6.51 , d.f. $=12, \mathrm{p}=0.89$ ). On the other hand, model 4 that has the same effect for the recurrent events and the same baseline function had the largest AIC and $\mathrm{BIC}$ values, suggesting that this model had the poorest fit to the data. Therefore, model 2 was considered to be the best model.

\section{Same effect model}

Table 3 shows the significant risk factors and the estimated hazard ratios and 95\% confidence intervals as specified in model 2. Specifically, compared with patients in the placebo group, patients in the pravastatin group had a $29 \%(95 \%$ CI $17-39 \%)$ reduction in the risk for the both MI events. For one unit (mmol/l) increase in total cholesterol level, the risk of an MI event (both the first and second event) increased by $19 \%$

Table 2 Comparison of different semi-parametric models in the LIPID study

\begin{tabular}{lcccc}
\hline Model & Log-likelihood & d.f. & AIC & BIC \\
\hline Different baseline hazard & & & & \\
$\quad$ Model 1: Different effect & -7317.331 & 24 & 14682.66 & 14868.61 \\
$\quad$ Model 2: Same effect & -7320.586 & 12 & 14665.47 & 14758.14 \\
Same baseline hazard & & & & \\
$\quad$ Model 3: Different effect & -7646.675 & 24 & 15341.35 & 15527.29 \\
$\quad$ Model 4: Same effect & -7915.245 & 12 & 15854.49 & 15947.46 \\
\hline
\end{tabular}

$\mathrm{AIC}=$ Akaike Information Criterion; BIC = Bayesian Information Criterion; d.f. $=$ degree of freedom
Table 3 Same effect model of the recurrent MI events in the LIPID study

\begin{tabular}{|c|c|c|c|}
\hline Risk factor & Hazard ratio & $95 \% \mathrm{Cl}$ & P-value \\
\hline Age (year) & 1.02 & $1.01-1.03$ & $<0.001$ \\
\hline \multicolumn{4}{|l|}{ Smoking status } \\
\hline Non-smoker & 1.0 & & \\
\hline Current smoker & 1.51 & $1.20-1.90$ & $<0.001$ \\
\hline Total cholesterol & 1.19 & $1.09-1.30$ & $<0.001$ \\
\hline HDL cholesterol & 0.38 & $0.26-0.54$ & $<0.001$ \\
\hline \multicolumn{4}{|c|}{ Qualifying event for ACS } \\
\hline Unstable angina & 1.0 & & \\
\hline Single MI & 1.29 & $1.06-1.57$ & 0.01 \\
\hline Multiple Ml & 1.98 & $1.57-2.50$ & $<0.001$ \\
\hline \multicolumn{4}{|l|}{ Revascularization } \\
\hline Never & 1.0 & & \\
\hline Before ACS & 1.59 & $1.26-2.01$ & $<0.001$ \\
\hline Since ACS & 0.70 & $0.58-0.85$ & $<0.001$ \\
\hline \multicolumn{4}{|l|}{ History of stroke } \\
\hline No & 1.0 & & \\
\hline Yes & 1.47 & $1.09-1.99$ & 0.01 \\
\hline \multicolumn{4}{|l|}{ Diabetes mellitus } \\
\hline No & 1.0 & & \\
\hline Yes & 1.37 & $1.08-1.72$ & 0.009 \\
\hline \multicolumn{4}{|l|}{ Angina grade } \\
\hline CCVS $<$ III & 1.0 & & \\
\hline CCVS $\geq \| I$ & 1.45 & $1.15-1.82$ & 0.001 \\
\hline \multicolumn{4}{|l|}{ Treatment } \\
\hline Placebo & 1.0 & & \\
\hline Pravastatin & 0.71 & $0.61-0.83$ & $<0.001$ \\
\hline
\end{tabular}

ACS = Acute Coronary Syndrome; CCVS = Canadian Cardiovascular Society Class of angina; $\mathrm{HDL}=$ High Density Lipoprotein; $\mathrm{MI}=$ Myocardial Infarction.

(95\% CI 9 - 30\%); while for one unit (mmol/l) increase in HDL-cholesterol, the risk of an MI event decreased by $62 \%$ (95\% CI $46-74 \%)$. Having a history of stroke increased the risk of an MI event by 47\% (95\% CI 9 99\%) and a history of diabetes increased the risk of an MI event by $37 \%$ (95\% CI $8-72 \%$ ).

Similarly, compared with patients with unstable angina as their qualifying event, the risk for an MI event during follow-up was increased by $29 \%$ (95\% CI $6-57 \%$ ) in those with a single MI event prior to randomization, while patients with multiple MI events prior to randomization had a 98\% (95\% CI 57 - 150\%) increased risk. Compared with patients without a history of coronary revascularization, those who had revascularization before randomization had a 59\% (95\% CI 26 - 101\%) increased risk for an MI event during follow-up; while patients who had revascularization after the qualifying event and before randomization had a 30\% (15 - 42\%) decreased risk. Having an angina grade III also increased the risk of an MI event during follow-up by $45 \%$ (95\% CI 15 $82 \%)$. Smoking also increased the risk of an $\mathrm{MI}$ event by $51 \%$ (95\% CI 20 - 90\%). 


\section{Different effect model}

Table 4 shows the significant risk factors and the estimates of hazard ratios and 95\% confidence intervals as specified in model 1 . This model has different covariate effects for the first and second recurrent MI events. Based on the Wald test [34], there was no significant difference in the effects of these risk factors between the first and second MI events ( $p$-values were between 0.076 and 0.91 in all cases). For example, compared with patients in the placebo group, patients assigned pravastatin had a $28 \%(95 \%$ CI $17-38 \%)$ reduction in the risk for the first MI and 34\% (95\% CI 1 - 57\%) reduction in the risk of a second MI. Compared with patients with unstable angina as their qualifying event, patients with multiple MIs prior to randomization had a 92\% (95\% CI $54-140 \%$ ) increased risk for a first MI event and 146\% (95\% CI $34-354 \%$ ) increased risk for a second MI during follow-up.

Similarly, compared with patients who never had revascularization, patients who had coronary revascularization before their qualifying acute coronary syndrome had 54\% (95\% CI 23 - 92\%) increased risk for a first MI and $102 \%$ (95\% CI $10-274 \%)$ increased risk for a second MI during follow-up. Other risk factors (smoking status, history of stroke or diabetes mellitus and angina grade) significantly increased the risk for the first but not the second MI event, probably due to the small number of observed second MI events, but in no case was there evidence of a different effect on first MI compared to a second MI (Table 4).

Figure 1 shows the cumulative risk of a specific MI event after randomization under model 2 . For ease of drawing this figure, baseline values of all significant categorical variables and centralized values of all significant continuous variables were used in model 2 . These values included non-smoking status, 60 years of age, total cholesterol level of $193 \mathrm{mg} / \mathrm{dl}(5.0 \mathrm{mmol} / \mathrm{l}), \mathrm{HDL}$ cholesterol level of $39 \mathrm{mg} / \mathrm{dl}(1.0 \mathrm{mmol} / \mathrm{l})$, angina grade III or less, previous unstable angina as the qualifying acute coronary syndrome at baseline and no history of revascularization, stroke or diabetes mellitus prior to randomization. The cumulative risk of the first MI event

Table 4 Different effect model of the recurrent MI events in the LIPID study

\begin{tabular}{|c|c|c|c|c|c|c|c|}
\hline \multirow[t]{2}{*}{ Risk factor } & \multicolumn{3}{|c|}{ First Ml event } & \multicolumn{3}{|c|}{ Second $\mathrm{Ml}$ event } & \multirow[t]{2}{*}{ Wald test ${ }^{\dagger}$} \\
\hline & $\mathrm{HR}$ & $95 \% \mathrm{Cl}$ & P-value & $\mathrm{HR}$ & $95 \% \mathrm{Cl}$ & P-value & \\
\hline Age (year) & 1.02 & $1.01-1.03$ & $<0.001$ & 1.04 & $1.01-1.06$ & 0.007 & 0.14 \\
\hline \multicolumn{8}{|l|}{ Smoking status } \\
\hline Non-smoker & 1.0 & & & 1.0 & & & \\
\hline Current smoker & 1.49 & $1.20-1.85$ & $<0.001$ & 1.71 & $0.94-3.12$ & 0.079 & 0.63 \\
\hline Total cholesterol & 1.18 & $1.08-1.28$ & $<0.001$ & 1.34 & $1.08-1.66$ & 0.007 & 0.21 \\
\hline HDL cholesterol & 0.41 & $0.29-0.59$ & $<0.001$ & 0.17 & $0.06-0.47$ & 0.001 & 0.076 \\
\hline \multicolumn{8}{|c|}{ Qualifying event for ACS } \\
\hline Unstable angina & 1.0 & & & 1.0 & & & \\
\hline Single Ml & 1.27 & $1.06-1.53$ & 0.011 & 1.44 & $0.81-2.54$ & 0.21 & 0.66 \\
\hline Multiple Ml & 1.92 & $1.54-2.40$ & $<0.001$ & 2.46 & $1.34-4.54$ & 0.004 & 0.39 \\
\hline \multicolumn{8}{|l|}{ Revascularization } \\
\hline Never & 1.0 & & & 1.0 & & & \\
\hline Before ACS & 1.54 & $1.23-1.92$ & $<0.001$ & 2.02 & $1.10-3.74$ & 0.024 & 0.34 \\
\hline Since ACS & 0.70 & $0.58-0.85$ & $<0.001$ & 0.65 & $0.36-1.16$ & 0.14 & 0.76 \\
\hline \multicolumn{8}{|l|}{ History of stroke } \\
\hline No & 1.0 & & & 1.0 & & & \\
\hline Yes & 1.50 & $1.12-2.00$ & 0.006 & 1.31 & $0.57-3.04$ & 0.53 & 0.75 \\
\hline \multicolumn{8}{|l|}{ Diabetes mellitus } \\
\hline No & 1.0 & & & 1.0 & & & \\
\hline Yes & 1.40 & $1.12-1.75$ & 0.003 & 1.14 & $0.58-2.23$ & 0.70 & 0.53 \\
\hline \multicolumn{8}{|l|}{ Angina grade } \\
\hline CCVS $<$ III & 1.0 & & & 1.0 & & & \\
\hline CCVS $\geq I I I$ & 1.44 & $1.16-1.80$ & 0.001 & 1.48 & $0.83-2.65$ & 0.18 & 0.91 \\
\hline \multicolumn{8}{|l|}{ Treatment } \\
\hline Placebo & 1.0 & & & 1.0 & & & \\
\hline Pravastatin & 0.72 & $0.62-0.83$ & $<0.001$ & 0.66 & $0.43-0.99$ & 0.047 & 0.67 \\
\hline
\end{tabular}

† P-value for Wald test. ACS = Acute Coronary Syndrome; CCVS = Canadian Cardiovascular Society Class of angina; HDL = High Density Lipoprotein; $\mathrm{MI}=$ Myocardial Infarction. 


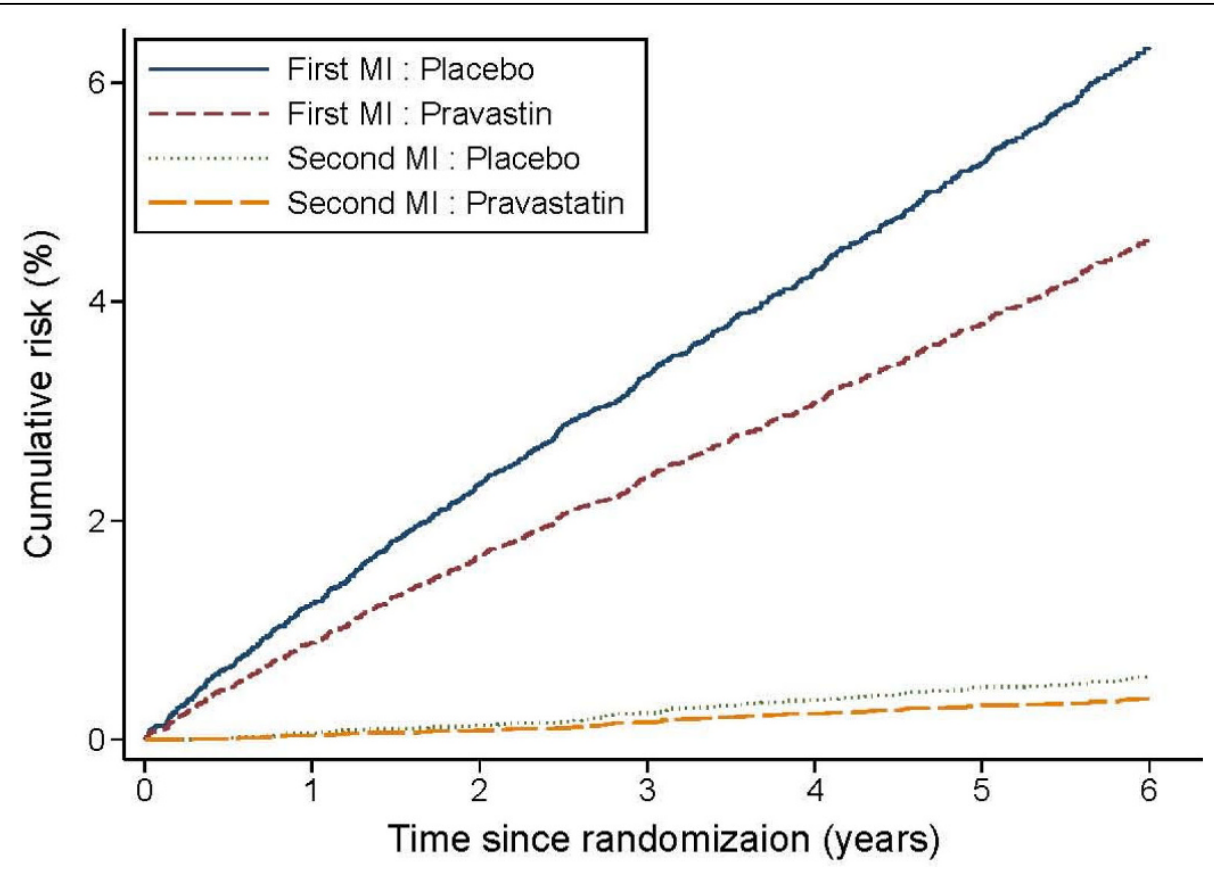

Figure 1 Estimated baseline cumulative risk of myocardial infarction (MI) during follow-up for a non-smoking patient aged 60 years with total cholesterol level $5.0 \mathrm{mmol} / \mathrm{l}, \mathrm{HDL}$ cholesterol $1.0 \mathrm{mmol} / \mathrm{l}$, angina grade less than III, with unstable angina as the qualifying acute coronary syndrome and no history of revascularization, stroke or diabetes mellitus before randomization.

within 5 years of randomization for such a person with these baseline values was $3.6 \%$ if the person was assigned to pravastatin and $5.1 \%$ if assigned to placebo. The cumulative risk for a second MI event within 5 years of randomization was lower but there was a similar relative treatment effect, being $0.5 \%$ for pravastatin and $0.7 \%$ for placebo.

\section{Same baseline hazard model}

We also fitted a semi-parametric model with the same baseline hazard function for the first and second MI events to the data. Compared with the estimated effects in Tables 3 and 4, the estimated effects under this new model increased slightly for the first MI event; while the estimated effects for the second MI event decreased slightly. The baseline cumulative risk in 5 years after randomization was about $2.8 \%$ for patients assigned to receive pravastatin and $3.6 \%$ for patients to receive placebo.

\section{First $\mathrm{Ml}$ event model}

Finally we fitted the traditional proportional hazards model by considering only the first MI event in the analysis, as shown in Table 5. Because the number of observations in this study was only half of those in Table 3 and 4 , this model was not comparable directly with those in Tables 3 and 4 using the AIC and BIC methods. However, the same significant explanatory variables in Tables 3 and 4 were found to be statistically significant for this model. The estimated effects of these risk factors were the same as those given in Table 4 for the first MI event.

\section{Model Comparison}

Compared with our previous analysis results under the parametric frailty model [10], we found that the same explanatory variables were selected into the final model in this study by the same selection procedure. The estimates of the regression coefficients and $95 \%$ confidence intervals were also similar. For example, the estimate of the smoking effect was 1.49 (95\% CI 1.17 - 1.89) under the parametric frailty model (Table 3 in [10]) compared with 1.51 (95\% CI 1.20 - 1.90) under the semi-parametric model (Table 3 in this article). Similarly, the estimate of the treatment effect of pravastatin was 0.71 (95\% CI $0.60-0.83)$ under the parametric frailty model compared with $0.71(95 \%$ CI $0.61-0.83)$ under the semi-parametric model.

However, interpretation of the risk of a recurrent event is different between the parametric conditional model and the semi-parametric proportional hazards model. The time period used to calculate the cumulative risk under the parametric conditional model was the time interval between two consecutive recurrent events; while it was from randomization to each recurrent event under the semi-parametric proportional hazards model. 


\begin{tabular}{|c|c|c|c|}
\hline Risk factor & Hazard ratio & $95 \% \mathrm{Cl}$ & P-value \\
\hline Age (year) & 1.02 & $1.01-1.03$ & $<0.001$ \\
\hline \multicolumn{4}{|l|}{ Smoking status } \\
\hline Non-smoker & 1.0 & & \\
\hline Current smoker & 1.49 & $1.20-1.86$ & $<0.001$ \\
\hline Total cholesterol & 1.18 & $1.08-1.29$ & $<0.001$ \\
\hline HDL cholesterol & 0.41 & $0.29-0.58$ & $<0.001$ \\
\hline \multicolumn{4}{|c|}{ Qualifying event for ACS } \\
\hline Unstable angina & 1.0 & & \\
\hline Single Ml & 1.27 & $1.06-1.53$ & 0.008 \\
\hline Multiple Ml & 1.92 & $1.55-2.39$ & $<0.001$ \\
\hline \multicolumn{4}{|l|}{ Revascularization } \\
\hline Never & 1.0 & & \\
\hline Before ACS & 1.54 & $1.24-1.90$ & $<0.001$ \\
\hline Since ACS & 0.70 & $0.58-0.85$ & $<0.001$ \\
\hline \multicolumn{4}{|l|}{ History of stroke } \\
\hline No & 1.0 & & \\
\hline Yes & 1.50 & $1.12-2.00$ & 0.007 \\
\hline \multicolumn{4}{|l|}{ Diabetes mellitus } \\
\hline No & 1.0 & & \\
\hline Yes & 1.40 & $1.12-1.74$ & 0.003 \\
\hline \multicolumn{4}{|l|}{ Angina grade } \\
\hline CCVS $<$ III & 1.0 & & \\
\hline CCVS $\geq \| I$ & 1.44 & $1.16-1.80$ & 0.001 \\
\hline \multicolumn{4}{|l|}{ Treatment } \\
\hline Placebo & 1.0 & & \\
\hline Pravastatin & 0.72 & $0.62-0.83$ & $<0.001$ \\
\hline
\end{tabular}

ACS = Acute Coronary Syndrome; CCVS = Canadian Cardiovascular Society Class of angina; $\mathrm{HDL}=$ High Density Lipoprotein; $\mathrm{Ml}=$ Myocardial Infarction.

For example, under the parametric conditional model (Figure 1 in [10]), the cumulative risk of a second MI event within 5 years was estimated to be $10.3 \%$ and $7.6 \%$ for persons assigned to placebo and pravastatin, respectively. However, under the semi-parametric proportional hazards model, the corresponding risk was estimated to be $0.7 \%$ and $0.5 \%$, respectively. It is noteworthy that the cumulative risk for the first MI event was similar under these two models because the common time origin was used in both methods $(5.6 \%$ and $4.1 \%$ under the parametric conditional model compared with $5.1 \%$ and $3.6 \%$ under the semi-parametric proportional hazards model).

\section{Discussion}

In this article, we applied several semi-parametric models [i.e., Wei, Lin and Weissfeld (WLW) models] to the analysis of the risk of recurrent myocardial infarctions in the LIPID study. We found that risk factors such as age, smoking status, total cholesterol and HDL cholesterol levels, the nature of the qualifying acute coronary syndrome, coronary revascularization, history of stroke or diabetes, angina grade and treatment with pravastatin were significant in the development of an MI event during follow-up. No significant difference was found in the effects of these risk factors between the first and second MI events. This is consistent with ongoing benefits of pravastatin for patients after they have a first MI while on active treatment. This analysis result is also consistent with a previous study [24], which analyzed data from the first MI and fatal coronary heart diseaserelated death only.

A major concern about the WLW model is that each individual is considered to be at risk of all recurrent events from the start of the observation period, while the conditional model [11] assumes that an individual is at risk of the $\mathrm{k}^{\text {th }}$ event only if the person experienced the $(\mathrm{k}-1)^{\mathrm{th}}$ event. However, the WLW method has been applied to recurrent event data because the nature of the relationship between recurrent events needs not to be known at the commencement of a study $[6,14]$. Furthermore, treatment effects are estimated based on the comparison of treatment and placebo groups. The WLW method is based on groups comparable at the time of randomization [35,36].

It has been suggested that a fuller picture of the treatment effect can be obtained from the application of both the marginal and conditional models [14,37]. We have published analysis results based on the conditional model [10]. One aim of this article is to compare the results of the marginal model with those from the conditional model.

Comparison of our previous findings with the current analysis shows that the semi-parametric models selected the same explanatory variables compared with the parametric conditional frailty models. The estimates of the regression coefficients and 95\% confidence intervals were also similar in the parametric and semi-parametric models. However, interpretation of the risk of a recurrent event is different between the parametric conditional model and the semi-parametric proportional hazards model. Under the parametric conditional model, the risk of a recurrent event is conditional on a person having had an MI event. The time period for calculating this risk is based on the gap between two consecutive recurrent events. This study shows that the chance of having two MI events within 5 years was low among all participants in the LIPID study; while the previous analysis suggested that the chance of having another MI event among a subset of participants who have already had an MI event was moderate.

Both semi-parametric proportional hazards model and the parametric conditional model are useful tools to further explore the biological process of a medical condition, such as different clinical manifestations of coronary atherosclerosis, our most common major health 
problem. They allow examination of the sequential occurrence of related events over time. The magnitude and direction of the impact of potential risk factors on these multiple events can also be examined using this approach. Additionally, the relative importance of the predictive values of a risk factor can be compared for different events. It is noteworthy that age, total cholesterol and HDL cholesterol were significant predictors of recurrent as well as the first myocardial infarction during follow-up.

The WLW method has been criticized because of its failure to accommodate the ordered nature of the recurrent events, that is, an individual can be at risk for the $\mathrm{k}^{\text {th }}$ event before the person experienced the $(\mathrm{k}-1)^{\mathrm{th}}$ event [38]. Essentially the WLW method assumes that the treatment effect on event $\mathrm{k}$ will be "carried over" to subsequent events, which may possibly cause biased estimates of the treatment effects for the later events [39]. According to the definition of the risk set of the WLW method, the occurrence of the first event also influences the time spent at risk for subsequent events though there is no direct treatment effect on the later events.

Another common criticism of the WLW method is that there is no simple joint distribution of all event times to satisfy the proportional hazards assumption [38]. Finally, the WLW method has been criticised for its failure to model the within-subject association of the recurrent events directly, which could possibly result in inefficient estimation of the treatment effect. However, this issue is balanced by the easy implementation of the robust variance estimates for any possible model misspecification.

Pravastatin has been shown to decrease coronary events in a number of studies. A similar reduction in risk of coronary heart disease death or nonfatal myocardial infarction was observed in the Cholesterol and Recurrent Events (CARE) study [40] and the LIPID study [22-24]. The first occurrence of a nonfatal MI or coronary heart disease death was considered in these analyses [24,41], although other endpoints have been included in other studies $[41,42]$. In this article, advanced statistical methods were applied to analyze the treatment effect of pravastatin for recurrent myocardial infarctions. The analyses suggest that the treatment effect of pravastatin was significant not only for the first MI event, but also the second MI event during followup. Furthermore the magnitude of the treatment effect was similar for first and second events.

There are some limitations in this study. Insufficient statistical power may have limited examination of the importance of the conventional risk factors. The nonsignificance of the effects of some risk factors for the second MI event could be related to the smaller number of second MI events during follow-up. Greater statistical power can be achieved by expanding the outcome to include other frequently observed endpoints, such as unstable angina or the need for coronary revascularization (percutaneous coronary intervention or coronary artery bypass graft surgery). Another potential limitation is that we did not consider the possibility of informative censoring of a fatal event which would preclude the occurrence of possible future MI events [2,4,39,43-45]. Joint modeling of recurrent events including a possible fatal event is needed in future investigation.

\section{Conclusions}

In conclusion, we found that the application of semiparametric proportional hazards model to the analysis of recurrent event data is informative and convenient, especially in the estimation of regression coefficients and cumulative risks. The treatment effect of pravastatin was similar on first and subsequent MI events. Important baseline characteristics showed no evidence of different predictive abilities between first and subsequent events.

\section{Author details}

'World Health Organization Collaborating Centre for Obesity Prevention, Deakin University, Melbourne, Australia. ${ }^{2}$ Department of Epidemiology and Preventive Medicine, Monash University, Melbourne, Australia. ${ }^{3} \mathrm{NHMRC}$ Clinical Trials Centre, University of Sydney, Sydney, Australia. ${ }^{4}$ Department of Statistics, Macquarie University, Sydney, Australia. ${ }^{5}$ Department of Medicine, University of Queensland, Brisbane, Australia.

\section{Authors' contributions}

$J C$ conducted the data analysis, interpreted the results and led drafting and revision of the manuscript. AF, AK and IM contributed to the planning of statistical analyses and contributed to the writing and revision of the paper AT, JS, DH and MW were involved in the LIPID study design, follow-up and analysis, and also involved in the conception and design of the present study, interpretation of results and revision and final approval of the manuscript.

\section{Competing interests}

The LIPID study was conducted under the auspices of The National Heart Foundation of Australia and funded by Bristol-Myers Squibb. AT, JS, AK, IM and MW were involved in the LIPID study.

Received: 6 May 2009 Accepted: 1 April 2010 Published: 1 April 2010

\section{References}

1. Nelson W: Recurrent Events Data Analysis for Product Repairs, Disease Recurrences, and Other Applications Philadelphia: American Statistical Association 2003.

2. Cook R, Lawless J: The statistical analysis of recurrent events New York: Springer 2007.

3. Hobson RW, Weiss DG, Fields WS, Goldstone J, Moore WS, Towne JB, Wright CB: Efficacy of carotid endarterectomy for asymptomatic carotid stenosis. The Veterans Affairs Cooperative Study Group. New England Journal of Medicine 1993, 328(4):221-227.

4. Li Q, Lagakos S: Use of the Wei-Lin-Weissfeld method for the analysis of a recurring and a terminating event. Statistics in Medicine 1997, 16:925-940.

5. Cox D: Regression models and life-tables. Journal of the Royal Statistical Society, Series B 1972, 34:187-220.

6. Wei L, Glidden D: An overview of statistical methods for multiple failure time data in clinical trials. Statistics in Medicine 1997, 16:833-839. 
7. Hougaard P: Analysis of Multivariate Survival Data New York: Springer-Verlag 2000.

8. Therneau T, Grambsch P: Modeling Survival Data Extending the Cox Model New York: Springer-Verlag 2000.

9. Cook R, Lawless J: Analysis of repeated events. Statistical Methods in Medical Research 2002, 11:141-166.

10. Cui JS, Forbes A, Kirby A, Marschner I, Simes J, West M, Tonkin A: Parametric conditional frailty models for recurrent cardiovascular events in the lipid study. Clinical Trials 2008, 5(6):565-574.

11. Prentice R, Williams $B$, Peterson A: On the regression analysis of multivariate failure time data. Biometrika 1981, 68:373-379.

12. Andersen $P$, Gill R: Cox's regression model for counting process: a large sample study. Annals of Statistics 1982, 10:1100-1120.

13. Wei $L$, Lin $D$, Weissfeld $L$ : Regression analysis of multivariate incomplete failure time data by modeling marginal distributions. Journal of the American Statistical Association 1989, 84:1065-1073.

14. Lin D: Cox regression analysis of multivariate failure time data: the marginal approach. Statistics in Medicine 1994, 13:2233-2247.

15. Pepe M, Cai J: Some graphical displays and marginal regression analyses for recurrent failure times and time dependent covariates. Journal of the American Statistical Association 1993, 88:811-820.

16. Lawless J, Nadeau J: Some simple robust methods for the analysis of recurrent events. Technometrics 1995, 37:18-68.

17. Glidden D, Vittinghoff E: Modelling clustered survival data from multicentre clinical trials. Statistics in Medicine 2004, 23:369-388.

18. Wang M-C, Chang S-H: Nonparametric estimation of a recurrent survival function. Journal of the American Statistical Association 1999, 94:146-153.

19. Pena E, Strawderman R, Hollander M: Nonparametric estimation with recurrent event data. Journal of the American Statistical Association 2001, 96:1299-1315.

20. Pena E, Slate E, Gonzalez J: Semiparametric inference for a general class of models for recurrent events. Journal of Statistical Planning and Inference 2007, 137:1727-1747

21. Metcalfe C, Thompson S: Wei, Lin and Weissfeld's marginal analysis of multivariate failure time data: should it be applied to a recurrent events outcome? Statistical Methods in Medical Research 2007, 16:103-122.

22. The Lipid Study Group: Design features and baseline characteristics of the LIPID (Long-Term Intervention with Pravastatin in Ischemic Disease) Study: a randomized trial in patients with previous acute myocardial infarction and/or unstable angina pectoris. American Journal of Cardiology 1995, 76:474-479.

23. The Lipid Study Group: The Lipid Study Group. Prevention of cardiovascular events and death with pravastatin in patients with coronary heart disease and a broad range of initial cholesterol levels. New England Journal of Medicine 1998, 339:1349-1357.

24. Marschner I, Colquhoun D, Simes R, Glasziou P, Harris P, Singh B, Friedlander $\mathrm{D}$, White $\mathrm{H}$, Thompson $\mathrm{P}$, Tonkin A: Long-term risk stratification for survivors of acute coronary syndromes. Results from the Long-term Intervention with Pravastatin in Ischemic Disease (LIPID) Study. Journal of the American College of Cardiology 2001, 38:56-63.

25. American Heart Association Medical/Scientific Statement: Revisions to classification of functional capacity and objective assessment of patients with diseases of the heart. Circulation 1994, 90(1):644-645.

26. Campeau L: Letter: Grading of angina pectoris. Circulation 1976, 54(3):522-523.

27. Cox D: Partial likelihood. Biometrika 1975, 62:269-276.

28. Lin $D$, Wei L: The robust inference for the Cox proportional hazards model. Journal of the American Statistical Association 1989, 84:1074-1178.

29. Huber P: The behavior of maximum likelihood estimates under nonstandard conditions. Proceedings of the Fifth Berkeley Symposium on Mathematical Statistics and Probability Berkeley: University of California Press $1967,1$.

30. White $\mathrm{H}$ : A heteroskedasticity-consistent covariance matrix estimator and a direct test for heteroskedasticity. Econometrica 1980, 48:817-830.

31. Kalbfleisch J, Prentice R: Statistical Analysis of Failure time Data New York: John Wiley \& Sons, 22002.

32. Stata Corporation: Stata Statistical Software: Release 10 College Station: Stata Corporation 2007.

33. Hollis $\mathrm{S}$, Campbell $\mathrm{F}$ : What is meant by intention to treat analysis? Survey of published randomised controlled trials. British Medical Journal 1999, 319:670-674.
34. Harrell FJ: Regression modelling strategies New York: Springer-Verlage 2001.

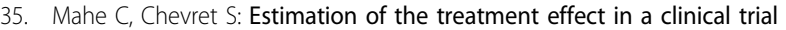
when recurrent events define the endpoints. Statistics in Medicine 1999, 18:1821-1829.

36. Ghosh D: Methods for analysis of multiple events in the presence of death. Control Clin Trials 2000, 21:115-126.

37. Barai U, Teoh N: Multiple statistics for multiple events, with application to repeated infections in the growth factor studies. Stat Med 1997, 16:941-949.

38. Cook R, Lawless J: Marginal analysis of recurrent events and a terminating event. Statistics in Medicine 1997, 16:911-924.

39. Kelly P, Lim L: Survival analysis for recurrent event data: an application to childhood infectious diseases. Statistics in Medicine 2000, 19:13-33.

40. Sacks FM, Tonkin AM, Shepherd J, Braunwald E, Cobbe S, Hawkins CM, Keech A, Packard C, Simes J, Byington R, et al: Effect of pravastatin on coronary disease events in subgroups defined by coronary risk factors: the Prospective Pravastatin Pooling Project. Circulation 2000, 102(16):1893-1900

41. Ridker PM, Buring JE, Rifai N, Cook NR: Development and validation of improved algorithms for the assessment of global cardiovascular risk in women: the Reynolds Risk Score. Journal of the American Medical Association 2007, 297:611-619.

42. Ishikawa J, Tamura Y, Hoshide S, Eguchi K, Ishikawa S, Shimada K, Kario K: Low-grade inflammation is a risk factor for clinical stroke events in addition to silent cerebral infarcts in Japanese older hypertensives: the Jichi Medical School ABPM Study, wave 1. Stroke 2007, 38:911-917.

43. Ghosh D, Lin D: Nonparametric analysis of recurrent events and death. Biometrics 2000, 56:554-562.

44. Wang M, Qin J, Chiang C: Analyzing recurrent event data with informative censoring. Journal of the American Statistical Association 2001, 96:1057-1065.

45. Liu L, Wolfe R, Huang X: Shared frailty models for recurrent events and a terminal event. Biometrics 2004, 60:747-756.

\section{Pre-publication history}

The pre-publication history for this paper can be accessed here: http://www. biomedcentral.com/1471-2288/10/27/prepub

doi:10.1186/1471-2288-10-27

Cite this article as: Cui et al: Semi-parametric risk prediction models for recurrent cardiovascular events in the LIPID study. BMC Medical Research Methodology 2010 10:27.

\section{Submit your next manuscript to BioMed Central and take full advantage of:}

- Convenient online submission

- Thorough peer review

- No space constraints or color figure charges

- Immediate publication on acceptance

- Inclusion in PubMed, CAS, Scopus and Google Scholar

- Research which is freely available for redistribution

Submit your manuscript at www.biomedcentral.com/submit
Biomed Central 